\title{
Habitat et société à Herculanum
}

Campagne 2014 du projet ANR VESUVIA

Alexandra Dardenay, Agnes Allroggen-Bedel, Hélène Eristov, Marie-Laure Maraval et Nicolas Monteix

\section{(2) OpenEdition \\ 12 Journals}

Édition électronique

URL : http://journals.openedition.org/cefr/1339

DOI : $10.4000 /$ cefr. 1339

ISSN : 2282-5703

Éditeur

École française de Rome

Référence électronique

Alexandra Dardenay, Agnes Allroggen-Bedel, Hélène Eristov, Marie-Laure Maraval et Nicolas Monteix, "Habitat et société à Herculanum », Chronique des activités archéologiques de l'École française de Rome [En ligne], Les cités vésuviennes, mis en ligne le 15 avril 2015, consulté le 01 mai 2019. URL : http:// journals.openedition.org/cefr/1339; DOI : 10.4000/cefr.1339

Ce document a été généré automatiquement le 1 mai 2019.

(C) École française de Rome 


\title{
Habitat et société à Herculanum
}

\author{
Campagne 2014 du projet ANR VESUVIA
}

\author{
Alexandra Dardenay, Agnes Allroggen-Bedel, Hélène Eristov, Marie-Laure \\ Maraval et Nicolas Monteix
}

\section{NOTE DE L'AUTEUR}

Notre reconnaissance la plus sincère est adressée aux institutions apportant leur collaboration au projet ANR VESUVIA (Vivre Ensemble. Société et Urbanisme d'une Ville de l'Italie Antique).

Cet article a été rédigé avec la collaboration de M. Carrive, A. Grand-Clément, N. Gautier, P. Mora et E. Rosso.

\section{Introduction}

1 Les campagnes 2014 (juillet et septembre) du programme VESUVIA (Vivre Ensemble. Société et Urbanisme d'une Ville de l'Italie Antique) ont permis de progresser significativement dans l'acquisition de données sur le site d'Herculanum, au Musée archéologique de Naples et dans les archives de la Soprintendenza di Pompei, Ercolano e Stabia. Parallèlement à l'enregistrement des décors et des données architecturales dans la base Domus $\subset$, nous avons pu avancer dans la restitution en contexte des peintures prélevées et déposées au Musée de Naples. La collaboration au projet des architectes et ingénieurs d'Archéovision (UMS SHS 3D n 3657 : Jean-François Bernard, Loïc Espinasse et Pascal Mora) est, à cet égard, particulièrement précieuse. Leur campagne d'acquisition de photographies sur le site leur permet actuellement de travailler sur les modèles 3D des édifices sélectionnés (cinq maisons en 2014). De ces modèles ont été extraites des orthophotographies sur lesquelles les membres de l'équipe peuvent projeter les restitutions de décors proposées à la suite de leurs investigations.

2 Ce travail d'analyse des données des précédentes campagnes et de réintégration des décors prélevés est toujours en cours. Nous avons choisi de commencer, pour 2014, par la 
Casa di Nettuno ed Anfitrite (V, 6-7) en raison du potentiel de restitution des décors de cette maison et d'une importante avancée de l'enquête grâce aux recherches menées dans les archives par Agnès Allroggen-Bedel. Le modèle 3D de la pièce $7 \mathrm{a}$ été récemment terminé (pour une première phase) par Pascal Mora, et permet donc de matérialiser nos hypothèses.

\section{Une étude des archives}

Des fouilles furent conduites dans cette maison sous la direction d'A. Maiuri entre novembre 1932 et mai 1934. Les journaux de fouilles (GSE) disponibles pour cette période donnent un compte rendu assez précis des dégagements réalisés dans cette maison et des découvertes qui y furent faites. Des disparités peuvent toutefois être constatées selon les journées de travail ou les pièces fouillées, le lieu de découverte de certains objets, notamment, étant parfois assez vaguement indiqué 1 . Lors de ces fouilles, les équipes d'A. Maiuri purent constater les dégâts commis dans la maison par les tunnels creusés sur ordre des Bourbons au XVIII ${ }^{e}$ siècle. Les GSE indiquent ainsi la destruction par un tunnel d'une grande partie du mur nord de la boutique (13), de l'escalier de la pièce 11 adjacente et du secteur sud-ouest de l'étage. Des dégâts furent également constatés dans le tablinum, où une partie du pavement d'opus sectile polychrome et des décors pariétaux manquaient, tout comme sur le mur est du nymphée (8). Deux tunnels condamnés étaient également observables dans le mur entre les pièces 5 et $6^{2}$. L'atrium ne fut pas non plus épargné par les spoliations des Bourbons : des parties du décor pariétal ont été prélevées, et peut-être également une partie du décor du laraire maçonné dans l'angle nord-ouest de cet espace. Ce laraire est mentionné par A. Maiuri comme étant très altéré au moment des fouilles qu'il mène dans la maison. Il présentait au moins deux tableaux sur marbre transportés au Musée de Naples, probables pendants de MN 9562, un autre tableau peint sur marbre trouvé le 24 mai 1746, qui présentait également la signature d'Athenaios ${ }^{3}$. La présence de ces tableaux sur marbre - sans doute réalisés à l'époque d'Auguste - faisaient de ce laraire un des exemples les plus originaux et les plus prestigieux connus à ce jour en Campanie ${ }^{4}$. Les dégradations occasionnées par les tunnels du XVIII ${ }^{e}$ siècle ont considérablement compliqué les fouilles de Maiuri, les équipes étant contraintes de consolider voire de reconstruire et de restaurer au fur et à mesure du dégagement. Un des tunnels a permis aux ouvriers du XVIII siècle de passer de la pièce 2 de la Casa dell'atrio corinzio ${ }^{5}$ (explorée en janvier-février 1746) au nymphée 8 de la Casa di Nettuno ed Anfitrite par le mur mitoyen et, de là, à la pièce 7 (explorée en mars 1746).

4 La Casa di Nettuno ed Anfitrite, telle qu'elle se présentait en 79 apr. J.-C., était le dernier état d'un édifice construit ex novo entre la fin du règne d'Auguste et le séisme de 62 apr. J.-C. ${ }^{6}$, sur un emplacement entièrement dégagé des structures préexistantes ${ }^{7}$. Cette maison fut apparemment épargnée à l'occasion des destructions que subit Herculanum en 62, mais connut d'assez importants dégâts dans la zone de l'atrium durant le « second séisme » qui se produisit à une date incertaine entre 70 et 75 apr. J.- ${ }^{8}$. En effet, l'effondrement du mur sud de la pièce 4 de la Casa del bel cortile $(\mathrm{V}, 8)$ a provoqué la destruction du mur nord, adjacent, de l'atrium de la Casa di Nettuno ed Anfitrite et sans doute d'une importante partie du compluvium (fig. 1). 
Fig. 1 - Herculanum, plan de l'insula V.

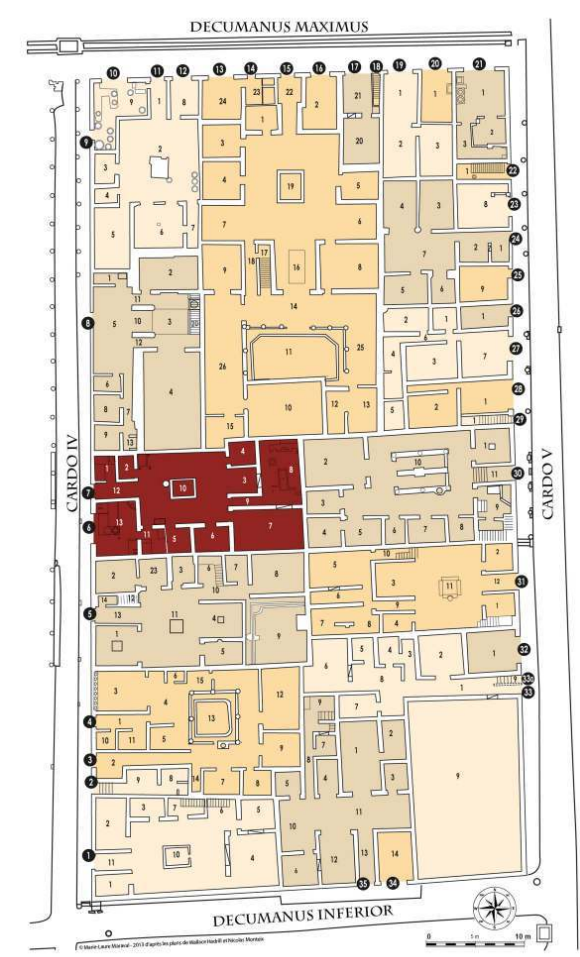

\section{EN ROUgE LA CASA DI NETTUNO ED ANfITRITE.}

Vectorisé par M.-L. Maraval d'après le plan de Monteix 2010 et Wallace-Hadrill 2011.

Il est même possible que les dégâts aient été plus importants, car la reconstruction fut associée à une phase de redécoration d'une partie, au moins, du rez-de-chaussée. La grande cohérence qui définit les décors de l'atrium (10), du tablinum (3) et du cubiculum (4), en particulier, apporte une unité profonde au programme décoratif de cette maison, entièrement ornée de peintures appartenant au IV ${ }^{e}$ style pompéien ${ }^{9}$ (fig. 2). 
Fig. 2 - Herculanum, Casa di Nettuno ed Anfitrite, plan du rez-de-chaussée.

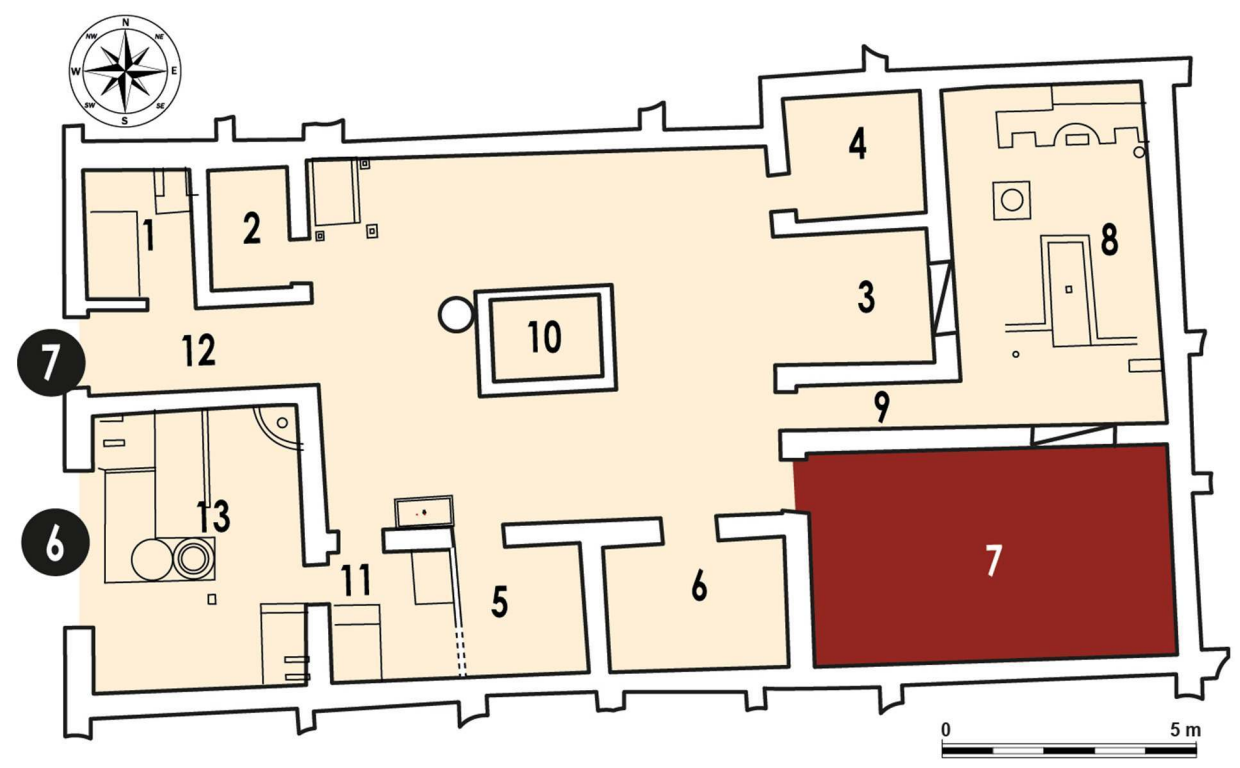

Vectorisé par M.-L. Maraval, d'après le plan de Monteix 2010 et Wallace-Hadrill 2011.

6 Entre sa construction à l'époque augustéenne et son état final, la maison a connu quelques modifications de son plan, mais pas de transformation majeure. Elle disposait d'une surface au sol de $197 \mathrm{~m}^{2}$, et de deux espaces à l'étage : l'un - situé dans la partie ouest de la maison (pièces 14-22), donnant sur la rue et pourvu d'une loggia - occupait environ $70 \mathrm{~m}^{2}$, et un second, aménagé au dessus du tablinum (pièces 23-24) ne couvrait que $16 \mathrm{~m}^{2}$ environ. La Casa di Nettuno ed Anfitrite était une maison à atrium de taille moyenne ${ }^{10}$. L'étage de la maison fait partie intégrante de son plan initial et fut construit en même temps que le rez-de-chaussée ce qui renforce l'impression d'unité architecturale procurée par l'édifice (fig. 3). 
Fig. 3 - Herculanum, Casa di Nettuno ed Anfitrite, plan de l'étage.

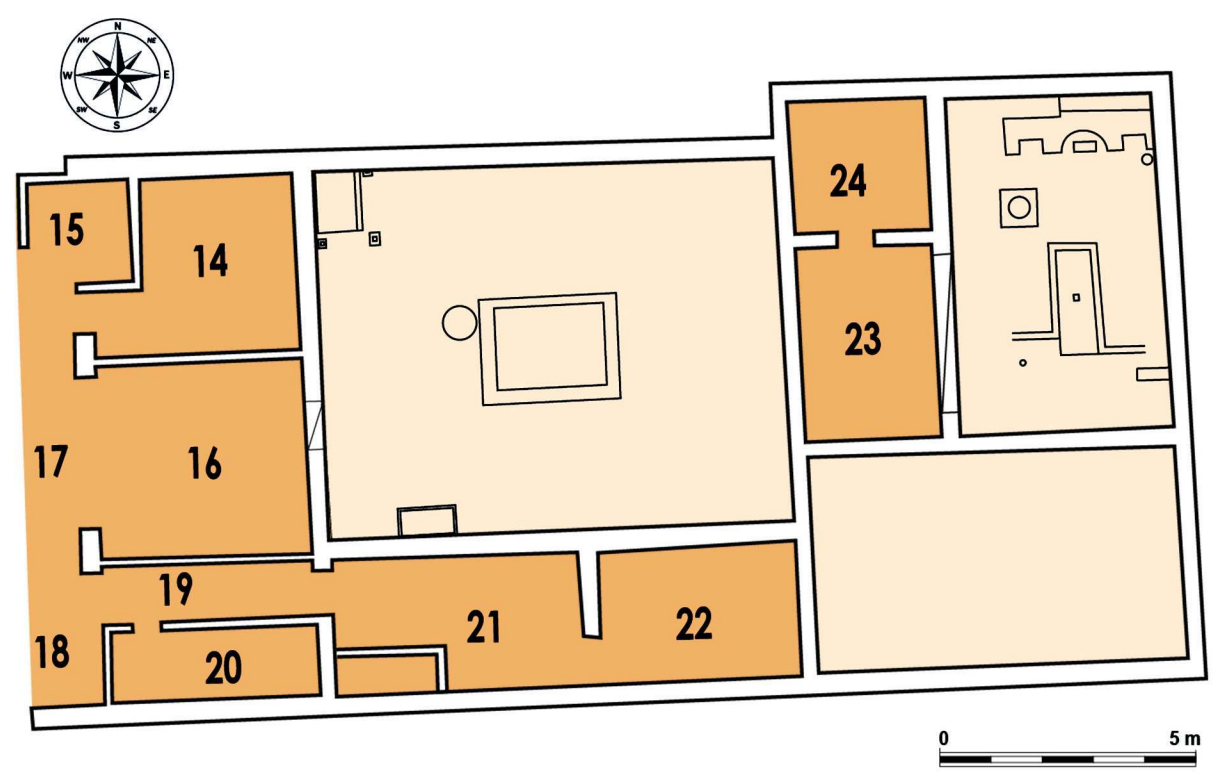

Vectorisé par M.-L. Maraval, d'après Andrews 2006 et Monteix 2010.

7 Les pièces d'habitations à l'étage de la partie ouest (14-22) étaient accessibles depuis un escalier en bois installé dans la pièce 11 tandis que le petit logement situé au dessus du tablinum (23-24) était sans doute accessible, au moins dans la phase finale, par une échelle amovible permettant d'accéder depuis le nymphée (8) à la pièce 23.

8 La maison fut donc l'objet d'une campagne unique de construction, dans la première moitié $\mathrm{du} \mathrm{I}^{\mathrm{er}}$ siècle, puis d'une campagne de restauration quelques années avant l'éruption. Une inscription peinte déchiffrée dans sur la paroi ouest de l'atrium, laisse d'ailleurs supposer que le décor de cet espace n'était pas entièrement achevé en 79 apr. J.C. En effet, l'étude des GSE a permis de relocaliser dans l'atrium de la Casa di Nettuno ed Anfitrite la mention d'une inscription peinte mentionnant la couleur " purpura» à appliquer sous le tableau à sujet mythologique du mur ouest, erronément attribuée à la Casa dell'alcova (IV, 3-4) dans le CIL ${ }^{11}$ (fig. 4 a et b). 
Fig. 4a et 4b - La paroi est de l'atrium (10) de la Casa di Nettuno ed Anfitrite.
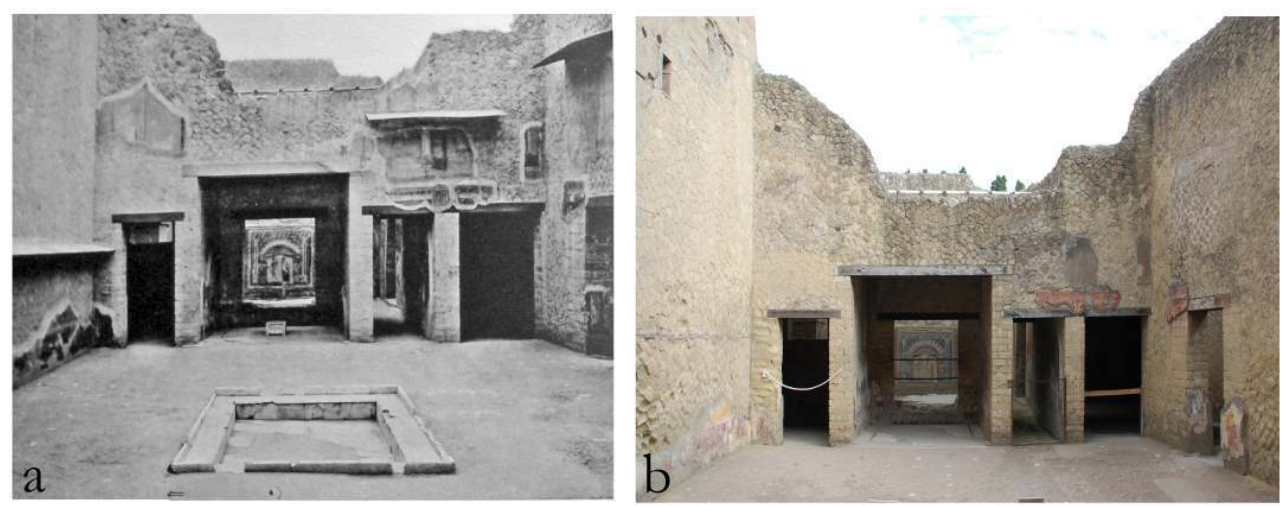

À droite, l'entrée de la pièce 7. Le cliché de gauche, paru dans Maiuri 1958 (fig. 332), permet de constater qu'une partie du décor peint de l'atrium était conservé et restauré après la fouille de cette maison. Le cliché de droite a été pris par Marie-Laure Maraval en 2011. Quasiment tout le décor peint de l'atrium a aujourd'hui disparu.

Maiuri 1958 et Marie-Laure Maraval.

Entre sa construction et l'éruption de 79 apr. J.-C., l'espace habitable de la Casa di Nettuno ed Anfitrite n'avait subi, a priori, qu'une légère amputation, lors la transformation en boutique de la pièce 13 , située en façade, à droite des fauces, probablement après le séisme de $62^{12}$. Pourvue d'un comptoir maçonné en équerre dans lequel étaient inclus deux dolia, et de deux tables de cuisson, l'une à l'extrémité nord de la pièce (avec un dispositif de chauffe-eau sous pression) et une autre à l'extrémité sud, cette boutique était sans doute un commerce alimentaire. Le côté est de la pièce était surmonté d'une mezzanine qui, dans la restitution proposée par Maiuri et toujours visible aujourd'hui, est un espace de stockage occupé par des amphores, mais qui aurait très bien pu servir d'espace de couchage $^{13}$. En 79 apr. J.-C., le lien entre la boutique et la maison était conservé, grâce à une porte dans son mur est qui donnait accès à la pièce 11. La boutique (13) était ornée d'un décor schématique à fond blanc et à encadrements de filets rouges qui appartenait à une phase antérieure à la transformation de cette pièce en boutique. La pièce n'avait donc pas été redécorée après son affectation à un usage commercial et ses peintures reflétaient donc un état de la pièce correspondant à un usage antérieur que l'on supposera de nature plutôt domestique. Il n'y avait en tout cas, a priori, aucun souci de corrélation, de la part du propriétaire, entre la fonction et le décor de cette boutique.

La Casa di Nettuno ed Anfitrite fait partie d'un groupe de cinq maisons que notre équipe a sélectionnées en raison de leur potentiel et de leur localisation afin d'en proposer une étude et une restitution 3D les plus exhaustives possibles dans le cadre de la première partie du programme ANR VESUVIA. Pour répondre à cet objectif, Pascal Mora de l'équipe Archéovision (CNRS) a opéré une couverture photographique de plus de 5000 photos lors de la campagne 2014. La réalisation des orthophotographies, des relevés photogrammétriques et des restitutions 3D est en cours (fig. 5). 
Fig.5 - Vue générale de la pièce 7.

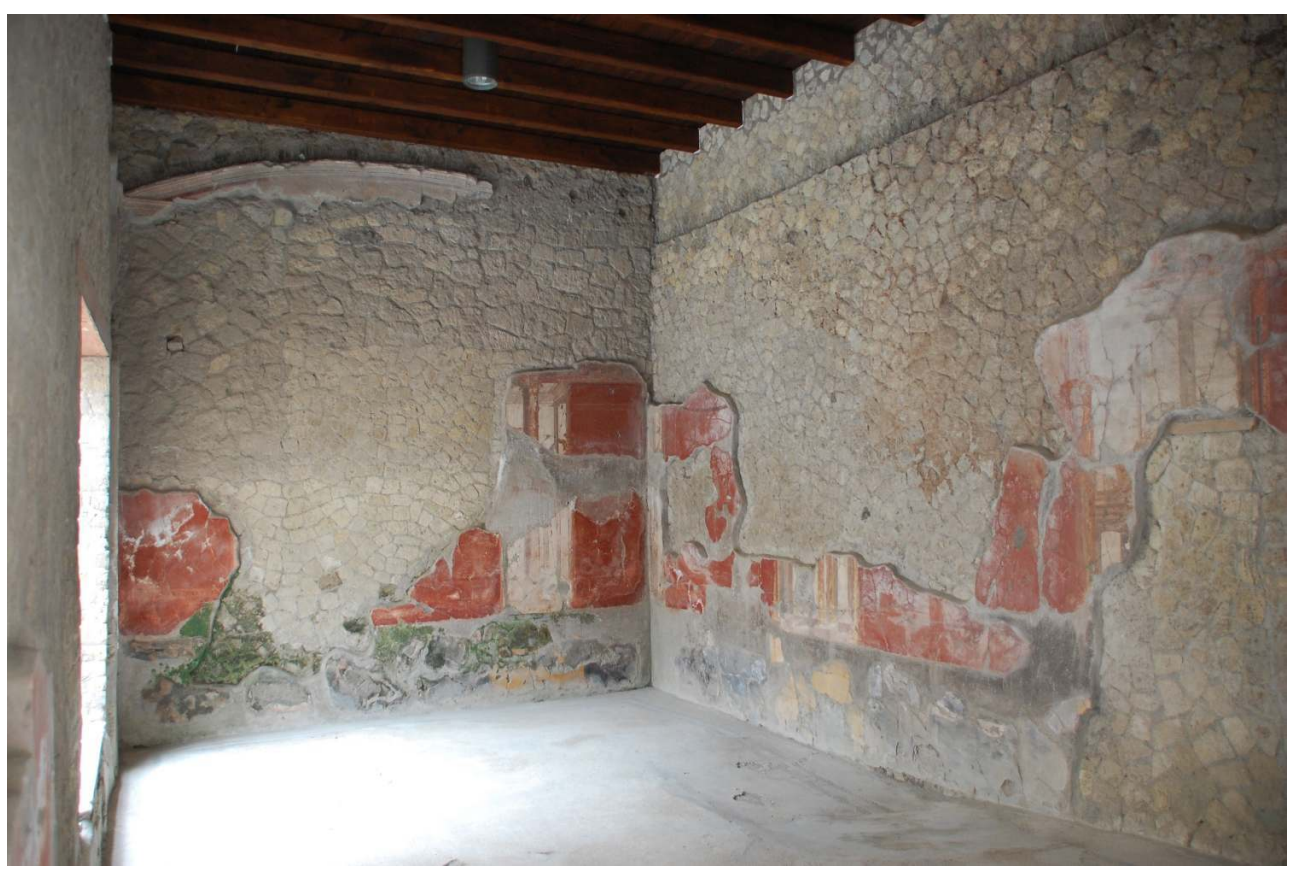

Au fond le mur est et à droite le mur sud.

Marie-Laure Maraval.

\section{De l'analyse des données à la restitution : l'exemple de la pièce 7}

11 L'architecture et le décor de la pièce 7 ont été l'objet d'une analyse particulièrement attentive lors de la campagne 2014. Cet espace d'imposantes dimensions (7,05 x 4,09 soit $28,83 \mathrm{~m}^{2}$ ), accessible depuis une porte ouvrant sur l'atrium (10), était couvert d'un plafond voûté culminant à $4,30 \mathrm{~m}$. Cette hauteur et l'architecture générale de la pièce nous assurent que ce triclinium n'était pas surmonté d'une pièce à l'étage, contrairement aux autres espaces de la maison (à l'exception de l'atrium bien entendu). Cette caractéristique nous confirme que les deux portions de l'étage ne communiquaient pas.

Par une large fenêtre ouverte dans le mur nord (et pourvue d'un volet de bois), la pièce 7 ouvrait sur le nymphée-triclinium (8) orné de la mosaïque pariétale éponyme de la maison ${ }^{14}$. Elle était pavée d'une mosaïque de tesselles blanches à double filet périmétrique noir, de facture soigneuse et contemporaine du IV style pictural ${ }^{15}$. Plusieurs caractéristiques indiquent que cette pièce, la plus grande de la maison, devait occuper une fonction de salle à manger et salle de repos (oecus/triclinium). En témoigne notamment son articulation avec le nymphée triclinium (8) qui révèle que toute la partie arrière de la maison était consacrée à l'otium. La découverte d'un fragment de banquette de bois dans cet espace ${ }^{16}$, seul vestige d'une pièce dépouillée d'une partie de son mobilier et de ses décors lors du passage des tunnels des Bourbons, en est un autre indice. 


\section{La fouille de la pièce 7 lors des nuovi scavi}

13 La pièce 7 n'est mentionnée que brièvement, entre le 8 et le 13 juin 1933, sans continuité, le 11 étant un dimanche, le 12 n'apportant aucune découverte mentionnée dans les journaux de fouilles. Les seuls objets mis au jour sont un fragment de panse d'amphore vinaire sicilienne portant une inscription peinte (le 8 juin), ainsi décrit lors de sa découverte :

[GSE 1933] 8 giugno. Nell'ambiente $\mathrm{N}^{\circ}$ 7, della Casa n. 6, posta sul IV Cardine, lato Est è stata raccolta una parte di vaso di terracotta con due iscrizioni fatte con pittura nera [suit un dessin de fiabilité douteuse reprenant l'inscription peinte].

14 Le même fragment d'amphore bénéficie de cette description dans les carnets de M. Della Corte, après autopsie lors de sa visite du 29 août 1933 :

Casa 6 - IV card[ine] Lato E[st] - ambiente 7. Resti di pancia di grossa anfora da un lato nero[:] a) [vinum] mol(lum) | A(uli) $G() C($ ) $\| I X[;]$ b) III alt[ezza m] 0.09. [au crayon bleu :] IV, 3-4.

15 Le seul autre objet mis au jour, mais non inventorié au moment de sa découverte, est un pied de lit en bronze, ensuite déplacé à l'étage lors de la mise en scène de la maison ${ }^{18}$.

Après la fouille, à l'exception des déplacements de fresque lors de la restauration, les transformations sont nulles, comme le confirme l'identité entre la description faite le 14 juin 1933 et celle rédigée une fois les restaurations effectuées, insérée à la fin du mois de juin 1934 :

[GSE 1934] Misura m. 7. 02 di lunghezza per m. 4. 05 di larghezza perciò è a forma rettangolare. Il vano è largo m. 1.20 per m. 1. 90 di altezza. La soglia è di tufo ed i pilastri di pietra tufo a filare. Tiene un secondo vano nella parete nord ed è largo m. 2 e alto m. 2.13 e piglia la luce da un triclinio scoperto e dall'atrio. La soglia è di marmo bianco della larghezza di m. 0.41. L'architrave di legno carbonizzato è di m. 0.13 di spessore ed i pilastri sono di pietra tufo a filare. Il pavimento è di tessere bianche con due piccole fasce poste quasi all'estremità. Poco stucco è sulle pareti ed è a fondo rosso e bianco con disegni di prospettive, colonne con capitelli e candelabri. Il soffitto è fatto ad incannucciata a tutto sesto di rosso e bianco. Tiene due cornici. Lo zoccolo è alto m. 0.70 ed è di stucco nero e giallo. La muratura è di opera incerta. È il più vasto ambiente della casa e poteva essere un triclinio invernale.

\section{La restitution du décor de la pièce 7 d'après les recherches d'Agnès Allroggen-Bedel}

Dans le triclinium 7, quelques vestiges du décor peint subsistent in situ et permettent d'attribuer à cette salle une série de peintures conservées au Musée archéologique de Naples ${ }^{19}$. Sur la zone supérieure du mur d'entrée (ouest), sous une petite lunette et une corniche de stuc, on voit, sur fond blanc une tenture rouge au bord richement orné. Devant cette tenture qui semble fixée à la corniche, apparaît le sommet d'un édicule avec un soffite à caissons reposant sur une colonnette dont le chapiteau corinthien est conservé.

18 Ces mêmes éléments se retrouvent sur une peinture conservée au Musée archéologique de Naples (9224, NR $112=$ PdE II. $34^{20}$ ). Il s'agit de la représentation d'un Hermaphrodite debout sous un édicule qui correspond parfaitement à celui resté in situ sur le mur ouest. 
Architecture, ornements et couleurs des deux édicules, parfaitement cohérents, attestent que la peinture à l'Hermaphrodite constituait le pendant du lambeau in situ et se situait sur le mur est dont le registre supérieur au-dessous de la lunette manque tout à fait; il n'est toutefois pas totalement exclu qu'il puisse provenir de l'un des longs murs (nord ou sud) de la pièce.

Les parentés formelles sont confirmées par les documents de fouille qui fournissent la la date du prélèvement et / ou de la découverte. Dans les rapports d'Alcubierre ${ }^{21}$, responsable des fouilles - „Noticias de las alajas...“ - comme aussi dans l'une de ses lettres adressées au Prince Salas, on lit :

05.03.1746 Alc. an Salas (ASN 1538)

Otra igual.te de 3 palmos la figura, representa muger desnuda, mas de medio cuerpo arriva; y lleva un belo, que procediendole de la caveza por la Espalda, sustiene porcion de el, con la mano drecha, y presentando un Tulipan con la izquierde, con parte del belo tiene cubiertas las piernas.

Comme il est fréquent, la description manque de précision et l'Hermaphrodite devient une figure féminine; cependant le geste de la main droite levant le voile, et celui de la main gauche tenant un flabellum sont bien identifiables.

21 Au Musée Archéologique de Naples il existe un pendant de l'Hermaphrodite : il s'agit d'une figure masculine sur fond blanc (MANN 8904 NR $224=$ PdE IV.10), représentant un jeune homme nu, à l'exception d'un drapé qui lui recouvre le bras gauche et la jambe droite, et la tête ceinte d'une couronne. Alcubierre mentionne également cette peinture :

21.03.1746

... La segunda, situada en medio de las otras dos, representa muger igual.te desnuda, de cuya mano izqui.a en que tiene un largo baston, con que apoya en tierra, le pende un pequeño paño, y estando situada, sobre una gran rueda, ó glovo, y coronada de ramos, pareze denota la fortuna.

De part et d'autre du tondo décrit par Alcubierre comme "una gran rueda, ó glovo », on distingue un entablement vu en contre-plongée. Il appartient à l'édicule central qui encadrait le panneau médian du mur ouest. La figuration de ce jeune homme au sceptre interprété par Alcubierre comme une figure féminine qu'il identifie comme "Fortuna » provient donc bien de la zone supérieure du mur ouest, où l'on voit encore le vide laissé par le prélèvement de la peinture.

Ces données nous indiquent donc avec certitude que, le 21 mars 1746, les fouilleurs se trouvaient dans le triclinium de la maison de Neptune et Amphitrite, à proximité du mur ouest.

Néanmoins une difficulté surgit; en effet, les deux lambeaux de décor « in situ » sur le mur ouest ne ménagent pas, dans leur situation actuelle, un espace suffisant pour repositionner le jeune homme au sceptre; afin d'aligner correctement le caisson de l'édicule médian visible sur MANN 8904 par rapport au reste de l'entablement « in situ », il faut d'une part baisser ce dernier, et d'autre part remonter le fragment de lunette, tous deux étant décalés dans la restauration. En effet, on sait que la technique adoptée par Maiuri consistait à consolider, voire à reconstruire, les murs antiques et à y replacer les enduits muraux, de sorte que les peintures in situ ne le sont pas réellement, comme cela apparait clairement dans le triclinium dont les enduits " en place» sont posés sur des murs manifestement modernes. Dans ce premier essai de restitution, tous les fragments in situ ont donc été déplacés, et la hauteur de la pièce semble légèrement supérieure à 
celle qui a été reconstruite. Ces hypothèses devront être validées lors d'une prochaine mission.

Pour compléter l'aspect originel de la pièce, d'autres peintures sont repérables grâce aux documents de fouille. Il s'agit d'une série de figures masculines nues, un drapé sur le bras : MANN 8899 NR $190=$ = PdE II.34 ; MANN 9369 NR $113=$ PdE II.35 ; MANN 8906 NR $188=$ PdE II.35 ; sans numéro d'inventaire, NR $193=$ PdE II.35. Debout, en pied, ces personnages sont couronnés de feuillages. Chacun d'eux tient un plateau d'une main, un rameau de l'autre. Du sommet de leur tête part une sorte de candélabre végétal qui amène à les identifier comme des atlantes. Ils semblent reposer sur une sorte de mensola à double volute, et se détachent devant une grande ouverture à fond blanc. L'un des fragments conserve la partie supérieure d'un entablement (NR 188), tandis que toute la partie supérieure manque sur un autre.

Ces figures sont signalées les 5 et 21 mars 1746 dans les rapports d'Alcubierre et accompagnées d'indications permettant de restituer leur emplacement. Le 5 mars 1746, il note :

La segunda, situada en medio de las otras dos, ...

27 La "deuxième peinture » désigne, dans le rapport, l'Hermaphrodite qui était donc originellement flanqué de deux figures masculines formant pendant, chacune avec un plateau et un rameau. De même, le jeune homme au sceptre détaché du mur ouest le 21 mars 1746 a été trouvé, lui aussi, avec deux figures de ce type, décrites à la même date : «hombre desnudo, gran plato en la mano drecha, ramo en la izquierda» et une autre « semejante á la primera, el plato en la mano izquierda y el ramo en la drecha ».

S'il est donc clair que les deux grandes figures de l'Hermaphrodite et du jeune homme au sceptre étaient encadrées des "atlantes ", il reste à tenter de préciser leur localisation exacte. Dans cette visée, plusieurs critères sont retenus. D'une part, la «mensola » qui constitue le sommet d'un support doit légitimement s'aligner sur l'un des supports de la zone médiane et il est très probable qu'il s'agit de la colonne de l'édicule médian visible sur le mur ouest. Comme, par ailleurs, sur ce même mur, la tenture rouge est accrochée à un membre architectural vertical dont on devine encore l'amorce in situ, une hypothèse vraisemblable voit dans ce support la limite du pavillon servant de cadre à l'atlante, et la tenture retomberait alors le long de cette architecture. D'autre part la largeur de l'ouverture à fond blanc diffère sur les différents fragments, et l'on tend à restituer les plus étroits sur les murs courts est et ouest, et les plus larges sur les longs murs nord et sud. Ainsi le jeune homme au sceptre du mur ouest pourrait être flanqué de MANN 9369 NR 113, à gauche, et de MANN 8906 NR 188, à droite. Quant à l'Hermaphrodite, il serait flanqué, à droite, de MANN 8899 NR 190, et à gauche d'une figure perdue. Par ailleurs MANN 9369 NR 113 semble situé dans un encadrement légèrement différent : la gravure des Pitture d'Ercolano (II.35) présente, à droite de la figure, un élément vertical qui semble cylindrique et diffère de l'encadrement des autres figures. Même si le panneau conservé au musée de Naples n'atteste pas clairement, en raison de son effacement, la présence d'une colonne, il reste que l'habituelle fidélité des graveurs de l'Accademia Ercolanese ne peut pas ne pas entretenir le doute et amener à penser que cette figure se situait sur l'un des longs murs, nord ou sud. 
Fig.6 - Orthophotographie du mur est de la pièce 7.

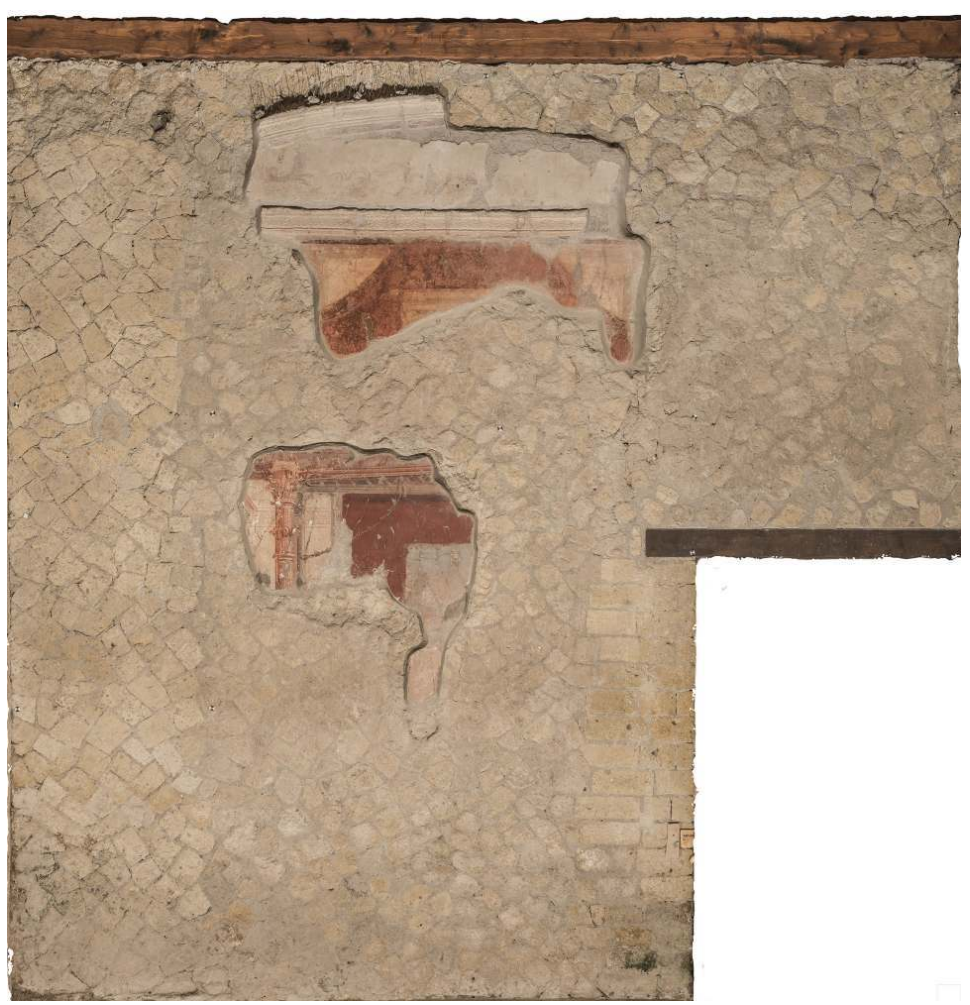

Pascal Mora (@Archéovision). 
Fig.7 - Sur l'orthophotographie (fig. 6), les fragments de peinture restaurés sous Maiuri sont repositionnés.

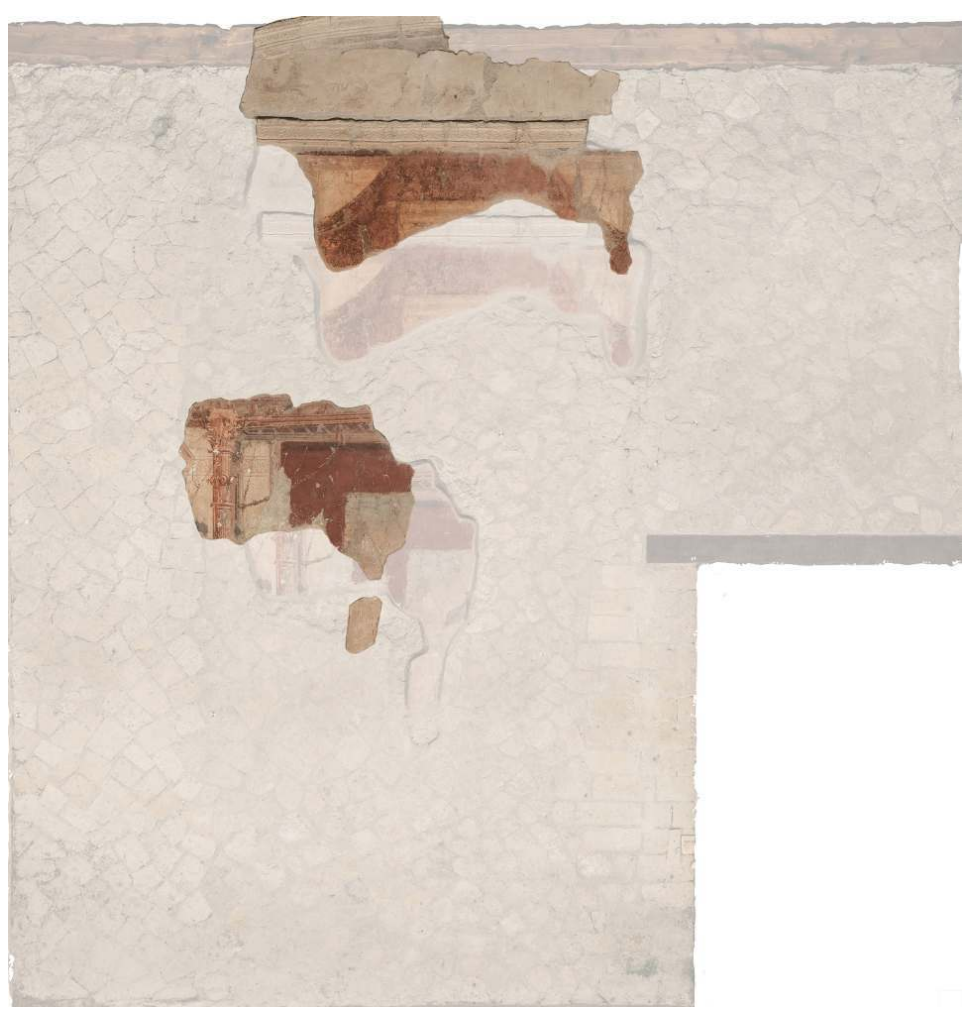

Infographie Marie-Laure Maraval.

Fig. 8 - Hypothèse provisoire de restitution infographique du décor de la zone haute du mur est de la pièce 7 .

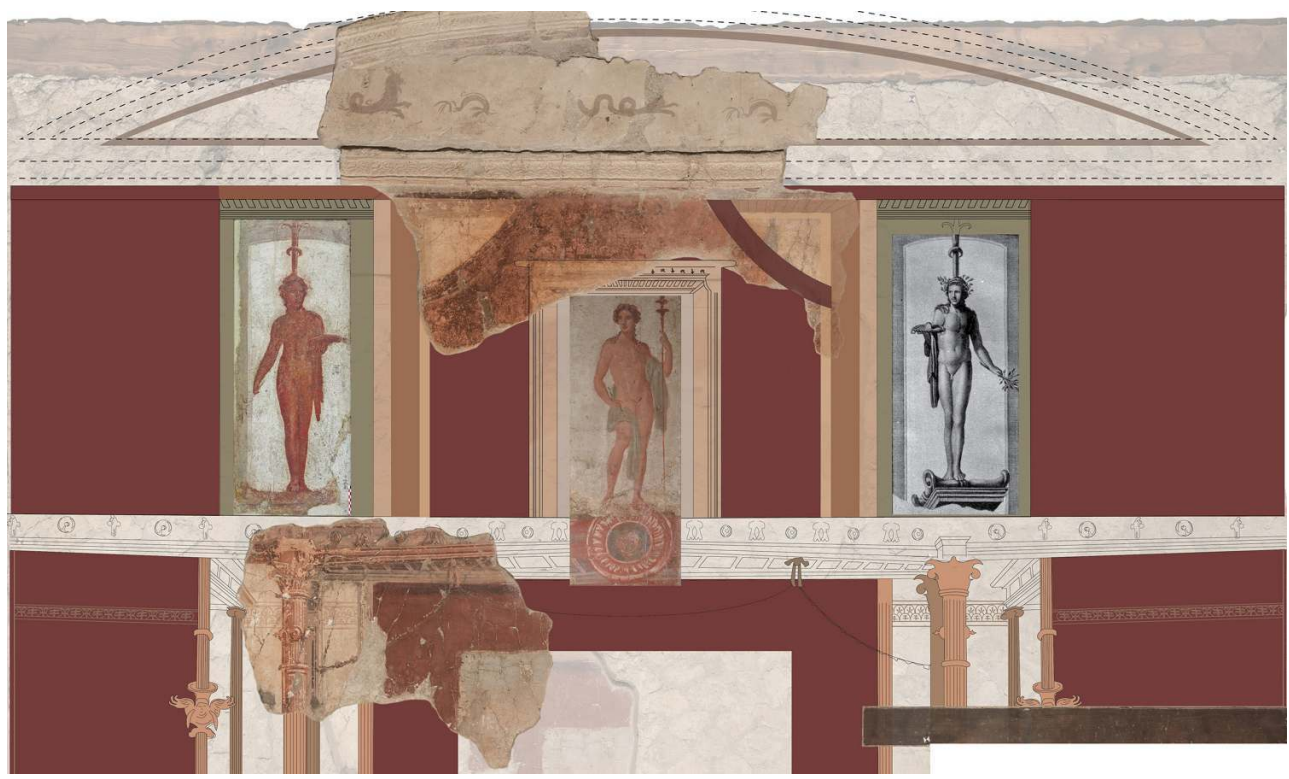

Infographie A. Allroggen-Bedel, H. Eristov et M. L. Maraval.

Pour restituer l'aspect de la zone haute de ces longs murs, les données sont très lacunaires. On sait, par les vestiges in situ, que le champ rouge central, encadré d'un 
édicule, était flanqué de deux échappées architecturales à fond blanc dont l'aspect est restituable puisque, sur le mur sud, celle de gauche a conservé sa partie inférieure, et celle de droite sa partie supérieure : à deux niveaux, elles étaient articulées par une paire de colonnes torsadées en plan fuyant, délimitant vers l'extérieur une ouverture large où apparait un personnage, et vers le centre une ouverture plus étroite séparée de l'édicule central par une colonne; au niveau supérieur une large baie couverte d'un plafond à solives reposant sur une grosse colonne évasée en large ombelle abrite une sorte de bétyle avec des objets suspendus. Dans ce dispositif, NR 193 pourrait être restitué audessus de l'échappée gauche, au droit de la colonne qui divise l'échappée ; ainsi la baie à fond blanc de l'atlante prolongerait celle de l'échappée, et il se trouverait dans une situation comparable à ceux des murs est et ouest. Mais dans la mesure où les vestiges à ce niveau sont peu lisibles ou disparus, cette hypothèse ne peut être vérifiée ; de plus, les extrémités des longs murs nord et sud comportent, elles aussi, des échappées architecturales plus étroites éventuellement surmontées de figures.

En revanche, nous disposons d'un élément supplémentaire pour restituer le décor de la zone médiane des murs nord et sud. Le rapport d'Alcubierre à la date du 25 février 1746 donne la description d'une peinture correspondant au tableau représentant Phèdre et Hippolyte (MANN 9041, PdE III.15). Qu'il provienne du triclinium de la maison de Neptune et Amphitrite est probable en raison de la date de la trouvaille, mais il n'appartient ni au mur ouest où l'angle supérieur gauche d'un tableau subsiste encore, ni au mur est car la hauteur du champ rouge médian conservé ne permet pas de le positionner. Il pourrait trouver place au centre des murs nord ou sud. Cependant, la même difficulté, déjà rencontrée sur le mur ouest, se reproduit ici : dans l'état actuel du décor in situ sur le mur sud, un grand fragment rouge, visiblement non jointif, a été inséré en bas à droite du champ médian. Il empêche tout positionnement du tableau sur ce mur, et par conséquent aussi sur le mur nord dont le schéma est nécessairement le même. Connaissant les pratiques en usage au temps des fouilles de Maiuri, on peut légitimement supposer que ce grand fragment a été mal resitué, et que le tableau se trouvait au centre du mur. Toutefois la question de la hauteur des tableaux se pose. Il semble que les tableaux des murs est et ouest avaient une hauteur moindre ( $50 \mathrm{~cm}$ au maximum) que celle du tableau avec Phèdre et Hippolyte afin de s'insérer dans la surface disponible du champ rouge ; or l'angle de tableau in situ sur le mur ouest comporte lui-même deux restaurations successives : lors de la première (visible sur des clichés des années 70), on lui a ajouté un fragment presque jointif, lors de la seconde, ce fragment a été replacé nettement plus bas, ce qui fait excéder au tableau la hauteur requise. Une série de doutes subsiste donc et devra être éclaircie lors d'une prochaine mission.

31 Malgré ces difficultés qui tiennent aux conditions particulières des fouilles d'Herculanum, la triple enquête menée sur le site, dans les réserves du musée et dans les archives, permet de redonner à ce triclinium une bonne partie de son décor originel. Par ailleurs, cette étude de la recontextualisation générale des peintures, articulée à l'analyse du bâti, nous permettra prochainement de restituer la hauteur originelle des murs et de la voûte. 


\section{BIBLIOGRAPHIE}

Archivio di Stato, Napoli, Casa Reale Antica, 1538, fol. 20. 23. 24 = Pagano 2005, p. 66-68.

Biblioteca della Società Napoletana di Storia Patria, MS XX.B.19bis : Noticia de las Alajas que se han descubierto en las escavaciones de Resina, y otras ; en las diez y ocho años, que han corido desde 22 de Octubre de 1738, en que se empezaron, hasta 22 de Octubre 1756, que se van continuando. = Pannuti 1983, p. 233-234.

Allroggen-Bedel 1991 = A. Allroggen-Bedel, Lokalstile in der campanischen Wandmalerei, dans 4. Internationales Kolloquium Römische Wandmalerei. Köln, 20.-23. September 1989, Kölner Jahrbuch für Vorund Frühgeschichte, 24, 1991, p. 35-41.

Andrews $2006=\mathrm{J}$. Andrews, The Use and Developpement of upper Floors in Houses at Herculaneum [Thesis submitted for the Degree of Ph. D.Department of Archaeology. University of Reading, 2006]. Inédit.

Camardo 2006 = D. Camardo, Gli scavi ed i restauri di Amedeo Maiuri : Ercolano el'esperimento di una città museo, dans OCNUS, 14, 2006, p. 69-82.

Camardo - Notomista 2012 = D. Camardo, M. Notomista, Il « ninfeo » della Casa di Nettuno ed Anfitrite di Ercolano $(v, 7-6)$ : nuovi dati archeologici dai recenti lavori di restauro, dans Vesuviana, 2012, p. 157-198.

De Kind 1998 = R.E.L.B. de Kind, Houses in Herculaneum $:$ a new view on the Town Planning and the Building of Insulae III and IV, Amsterdam, 1998 (Circumvesuviana, 1).

Della Corte 1958 = M. Della Corte, Le iscrizioni di Ercolano, dans Rendiconti dell'Accademia di Archeologia, Lettere e Belle Arti di Napoli, 33, 1958, p. 239-308.

Esposito 2014 = D. Esposito, La pittura di Ercolano, Rome, 2014 (Studi della Soprintendenza archelogica di Pompei, 33).

Ganschow 1989 = Th. Ganschow, Untersuchungen zur Baugeschichte in Herculaneum, Bonn, 1989 ( Antiquitas, 30).

Guidobaldi et al. 2014 = F. Guidobaldi et al., Mosaici antichi in Italia, Regione Prima, Ercolano, PiseRome, 2014.

Lochin 2005 = Mosaïque et statue de culte : Neptune et Amphitrite à Herculanum, dans H. Morlier (éd.), La mosaïque gréco-romaine, Actes du colloque international pour l'étude de la mosaïque antique et médiévale, Rome, Italie, 5-10 novembre 2001, IX, Rome, 2005, p. 35-49.

Maiuri 1958 = A. Maiuri, Ercolano : i nuovi scavi (1927-1958), Rome, 1958.

Mols 1999 = S.T.A.M. Mols, Wooden Furniture in Herculaneum, Amsterdam, 1999 (Circumvesuviana, 2).

Monteix 2010 = N. Monteix, Les lieux de métier : boutiques et ateliers d'Herculanum, Rome, 2010 ( BEFAR, 344).

Pagano 2005 = M. Pagano, I primi anni degli scavi di Ercolano, Pompei e Stabiae $:$ raccolta e studio di documenti e disegni inediti, Rome, 2005 (Studi della Soprintendenza archeologica di Pompei, 11). 
Pannuti 1983 = U. Pannuti, Il « giornale degli scavi » di Ercolano (1738-1756), dans Atti della Accademia nazionale dei Lincei. Memorie della Classe di scienze morali, storiche e filologiche, 26, 1983, p. 163-410.

$\operatorname{PdE}[$ Pitture d'Ercolano] = Le pitture d'Ercolano e contorni incise con qualche spiegazione, I-V, Naples, 1757-1779.

Peña 2007 = J. T. Peña, Two groups of tituli picti from Pompeii and environs : Sicilian wine, not flour and hand-picked olives, dans JRA, 20, 1, 2007, p. 233-254.

Wallace-Hadrill 1994 = A. Wallace-Hadrill, Houses and society in Pompeii and Herculaneum, Princeton, 1994.

Wallace-Hadrill 2011 = A. Wallace-Hadrill, Herculaneum : Past and Future, Rome, 2011.

\section{NOTES}

1. Sur cette pratique à Herculanum, cf. Mols 1999, p. 26-30; Camardo 2006; Monteix 2010, p. 16-35.

2. Andrews 2006, p. 195.

3. Inv. 150210 (Femme trônant et jeune homme) et 150211 (deux femmes dansant près d'une statue de Poséidon). En bas à droite du tableau 150211 se trouve l'inscription : "Alexandre l'athénien m'a peint », même signature que sur le monochrome sur marbre MN 9562 (les joueuses d'osselet).

4. Ainsi que le souligne A. Maiuri (Maiuri 1958, p. 394)

5. Cf Chronique 2014 (Dardenay, Eristov, Maraval) : http://cefr.revues.org/1222.

6. La nouvelle maison fut construite complètement en opus reticulatum $B$ avec portes et montants de fenêtres en opus vittatum (Ganschow 1989, p.140, Andrews 2006, p. 177, Monteix 2010, p. 325-327).

7. La parcelle dévolue à cette habitation semble avoir été la même depuis la création de l'insula. De l'habitation antérieure (pré-augustéenne) rien n'a été conservé à l'exception de quelques traces d'opus incertum dans le mur périmétrique sud.

8. Monteix 2010, p. 327.

9. À l'exception de la boutique. Esposito 2014, p. 81-82.

10. Pour la classification des maisons d'Herculanum, plusieurs typologies ont été proposées, toutes assez insatisfaisantes, du point de vue même des auteurs. La plus ancienne est celle de Maiuri (1958), révisée par Wallace-Hadrill (1994) et de Kind (1998). Aucune d'entre elle ne prend en compte les étages, comme le souligne à juste titre J. Andrews (Andrews 2006, p. 94-96)

11. CIL, IV, 10480. Cf. Monteix 2010, p. 330, n. 72. D'après les GSE, cette inscription aurait été découverte le $1^{\mathrm{er}}$ mars 1933 : « 1 marzo. [...] Nella casa $\mathrm{N}^{\circ} 6$ sul IV cardine, lato est, nella parete ovest sopra un riquadro di un soggetto mitologico vi è la seguente iscrizione fatta con pittura : [dessin de l'inscription sans respecter sa forme en équerre, attestée sur le dessin exécuté par $\mathrm{F}$. Ferrajoli (Archivio disegni SANP, Pompei, P658 fo9)] È lunga m.0.085 e le lettere sono alte m. 0.03 ». Les carnets de M. Della Corte donnent la description suivante : « Casa 6 - Cardo IV lato $\mathrm{E}[\mathrm{st}$ - Amb(iente) 3 - Parete $\mathrm{O}$ [vest] Nella cornice $\mathrm{d}$ [estra] in alto di un grande quadro leggesi in col[ore] marrone PVR|PVRA [dessin de l'inscription suivant sa forme en équerre] » (Getty Research Institute, H. B. Vander Poel Campanian collection, I.C, box 51, 6, carnet $n^{\circ} 45,20$ mars 1933).

12. Monteix 2010, p. 389.

13. Pour l'inventaire des objets mis au jour dans cette pièce : Monteix 2010, p. 390. Sur la fonction de cette mezzanine, Andrews 2006, p. 185 sq. Par ailleurs, les GSE ne mentionnent pas la découverte d'amphores sur la mezzanine. 
14. Mosaïque qui d'après l'interprétation de C. Lochin ne représente pas Neptune et Amphitrite mais Neptune et Vénus (Lochin 2005). Sur l'étude du bâti : Camardo - Notomista 2012

15. Guidobaldi et al. 2014, p. 286.

16. Mols 1999, cat n8, p. 159-160.

17. Getty Research Institute, H.B. Vander Poel Campanian collection, I.C, box 51, 6, carnet $\mathrm{n}^{\circ} 46$, 29 août 1933. La mention au crayon bleu correspond à l'interprétation de la localisation telle qu'effectuée par M. Della Corte (dans la Casa dell'alcova, IV, 3-4) lors de la publication des inscriptions d'Herculanum (Della Corte 1958). Cette interprétation, erronée, a été suivie par Pio Ciprotti dans le fascicule consacré à Herculanum dans le supplément 3 du CIL IV (cf. CIL IV, 10753 a-b). Le développement proposé ici suit les interprétations de J. Th. Peña (2007). On notera à sa suite que cette inscription sur amphore vinaire se retrouve à l'identique à Pompéi (CIL IV, 5745).

18. Les GSE signalent la découverte du pied de lit dans la pièce 7 de la Casa di Nettuno e Anfitrite le 9 juin 1933. A. Maiuri (1958, p. 401-402) localise quant à lui un lit à l'étage. D'après S. Mols (1999, p. 31), c'est au terme de la fouille que ce lit a été disposé à l'étage.

19. Allroggen-Bedel 1991, p. 37-38 n. 28.

20. $\mathrm{NR}=$ numero romano. Il s'agit des numéros d'inventaire du XVIII ${ }^{\mathrm{e}}$ siècle.

21. Archivio di Stato, Napoli, Casa Reale Antica, 1538, fol. 20. 23. $24=$ Pagano 2005, p. 66-68. Biblioteca della Società Napoletana di Storia Patria, MS XX.B.19bis: Noticia de las Alajas que se han descubierto en las escavaciones de Resina, y otras; en las diez y ocho años, que han corido desde 22 de Octubre de 1738, en que se empezaron, hasta 22 de Octubre 1756, que se van continuando = Pannuti 1983, p. 233-234.

\section{INDEX}

institutions Soprintendenza speciale per i beni archeologici di Pompei, Ercolano e Stabia, Ufficio Scavi di Ercolano, Musée archéologique national de Naples, École française de Rome, Centre Jean Bérard, CNRS, ANR, ENS, Université Toulouse Jean Jaurès

Mots-clés : Herculanum, urbanisme romain, architecture, habitat, décor antique, peinture murale

\section{AUTEURS}

\section{ALEXANDRA DARDENAY}

Université Toulouse II Jean Jaurès, UMR 5608 « TRACES » (CNRS / Université de

Toulouse II) - adardenay[at]yahoo.fr

AGNES ALLROGGEN-BEDEL

riess-ab[at]t-online.de

HÉLÈNE ERISTOV

UMR 8546 «AOROC » (CNRS / ENS) - helene.eristov[at]ens.fr 


\section{MARIE-LAURE MARAVAL}

Université Toulouse II Le Mirail, DAR (Direction Appui Recherche), UMR 5608 « TRACES » (CNRS / Université de Toulouse II) - mmaraval[at]univ-tlse2.fr

\section{NICOLAS MONTEIX}

Université de Rouen, GRHis (EA 3831) - nicolas.monteix[at]univ-rouen.fr 